\title{
A sequence of nitrogen-rich very red giants in the globular cluster NGC $1851^{\star}$
}

\author{
Eugenio Carretta ${ }^{1}$, Valentina D’Orazi ${ }^{2,3}$, Raffaele G. Gratton ${ }^{4}$, and Sara Lucatello ${ }^{4}$ \\ 1 INAF - Osservatorio Astronomico di Bologna, via Ranzani 1, 40127 Bologna, Italy \\ e-mail: eugenio.carretta@oabo.inaf.it \\ 2 Dept. of Physics and Astronomy, Macquarie University, Sydney, NSW, 2109 Australia \\ 3 Monash Centre for Astrophysics, School of Mathematical Sciences, Building 28, Monash University, VIC 3800, Australia \\ ${ }^{4}$ INAF - Osservatorio Astronomico di Padova, Vicolo dell'Osservatorio 5, 35122 Padova, Italy
}

Received 11 November 2013 / Accepted 8 January 2014

\begin{abstract}
We present the abundances of $\mathrm{N}$ in a sample of 62 stars on the red giant branch (RGB) in the peculiar globular cluster NGC 1851. The values of $[\mathrm{N} / \mathrm{Fe}]$ ratio were obtained by comparing the flux measured in the observed spectra with that from synthetic spectra for up to about 15 features of $\mathrm{CN}$. This is the first time that $\mathrm{N}$ abundances are obtained for such a large sample of RGB stars from mediumresolution spectroscopy in this cluster. With these abundances, we provide chemical tagging of the split RGB found from several studies in NGC 1851. The secondary reddest sequence on the RGB is populated almost exclusively by N-rich stars, confirming our previous suggestion based on Strömgren magnitudes and colours. These giants are also, on average, enriched in s-process elements such as $\mathrm{Ba}$, and are likely the results of pollution from low-mass stars that experienced episodes of third dredge-up in the asymptotic giant branch phase.
\end{abstract}

Key words. stars: abundances - globular clusters: individual: NGC 1851 - stars: Population II - globular clusters: general stars: atmospheres

\section{Introduction}

In the variegated landscape traced by multiple stellar populations in globular clusters (GCs; see Gratton et al. 2004, 2012a, for recent reviews that summarize decades of studies on this topic), some objects present a further level of complexity.

This is the case of the globular cluster NGC 1851. Less massive than GCs where multiple main sequences were discovered for the first time (such as $\omega$ Cen, Anderson 1998; and NGC 2808, D'Antona et al. 2005), this cluster contained evidence of multimodality, starting with the bimodal distribution of stars on its horizontal branch (HB; Walker 1992) and following with the finding of a split subgiant branch (SGB). The origin of this feature is still debated, which possibly invokes explanations of age or CNO-content differences (Milone et al. 2008, 2009; Cassisi et al. 2008; Gratton et al. 2012b). Beside these features, a split in the red giant branch (RGB) was recently detected by Han et al. (2009) with UVI Johnson broadband photometry and by Lee et al. (2009a) using narrow band Ca II and Strömgren photometry. Side by side to a main RGB, another sequence of cluster red giant stars, less populated, is seen in these studies, and it recently was shown to be connected to the faint SGB by Lardo et al. (2012), using Strömgren photometry.

Three effects may produce a redder sequence of red giants in a stellar population:

1. The metal content (in particular the abundance of the $\alpha$-elements) is higher in the redder sequence. This implies an increase in the number of free electrons, and as consequence of the opacity, giving a redder RGB.

* Based on observations collected at ESO telescopes under programme 188.B-3002.
2. The relative age of the two sequences is different with the bluest one being younger.

3. Enhanced $\mathrm{N}$, along with $\mathrm{C}$, may result in a redder sequence.

Lee et al. (2009b) inserted NGC 1851 in the sample used to devise an enrichment scenario for multiple populations in GCs where supernovae ( $\mathrm{SNe}$ ) also do contribute to the pollution of the intracluster gas. This scenario is ruled out in most clusters, due to the constancy of their $\alpha$-element content (Carretta et al. 2010a), but we recently found a contribution by core-collapse $\mathrm{SNe}$ in a fraction of RGB stars (Carretta et al. 2011) from the abundance analysis of $\mathrm{Ca}$ and other $\alpha$-elements in NGC 1851. Moreover, a spread in $[\mathrm{Fe} / \mathrm{H}]^{1}$, which was small but exceeding the observational errors, was detected in the same analysis, confirming earlier results by Yong and Grundahl (2008) and Carretta et al. (2010b). Metal-rich and metal-poor giants also show a different radial distribution with more metal-rich stars being less centrally concentrated (see Fig. 6 in Carretta et al. 2011); however, these stars are not segregated exclusively along the reddest RGB sequence.

A spread in the total CNO sum was found by Yong et al. (2009) in a limited sample of four RGB stars in NGC 1851. Enhanced CNO abundances in a fraction of stars were also proposed by Cassisi et al. (2008) as a possible explanation for the split SGB. The implication is that small mass asymptotic giant branch (AGB) stars (those experiencing episodes of third dredge-up) also contribute to the budget of nuclearly processed matter to construct a second generation of stars in NGC 1851.

1 We adopt the usual spectroscopic notation, which is for any given species $X,[X]=\log \epsilon(X)_{\text {star }}-\log \epsilon(X)_{\odot}$ and $\log \epsilon(X)=\log \left(N_{\mathrm{X}} / N_{\mathrm{H}}\right)+$ 12.0 for absolute number density abundances. 
Thus, a fraction of stars may be found to be younger than the other. However, Villanova et al. (2010) challenged this hypothesis in part by finding that the RGB stars on the two sequences do not show any significant difference in the total $\mathrm{C}+\mathrm{N}+\mathrm{O}$ sum but only in their content of s-process elements, in particular, for Ba. On the other hand, variations of s-process elements like $\mathrm{Zr}$ and $\mathrm{La}$ were found to be correlated to light elements (like Al) by Yong and Grundahl (2008): this again calls for contribution from thermally pulsing AGB stars of low initial masses.

Using a large set of elemental abundances for a large sample of giants, we recently proposed a classification scheme that divides the RGB stars in NGC 1851 into two populations using a combination of Fe and $\mathrm{Ba}$ (Carretta et al. 2011). Our data show that stars are nicely segregated along the split RGB according to their $\mathrm{Ba}$ abundances with Ba-poor stars located mostly (but not only) on the bluest side of the RGB, whereas only Ba-rich stars are found on the less populated reddest sequence. This result puts on a more statistically robust base similar findings by Villanova et al. (2010). We recently find evidence of a statistically significant correlation between $\mathrm{Al}$ and $\mathrm{Ba}$ abundances in the more metal-rich component of RGB stars (Carretta et al. 2012a).

Moreover, using the comparison of Strömgren colours and synthetic spectra computed with varying $\mathrm{C}$ and $\mathrm{N}$ abundances, we demonstrated that stars on the reddest sequence are very good candidates to be $\mathrm{C}$-rich and $\mathrm{N}$-rich, while stars that are $\mathrm{C}$-rich and N-poor (i.e. O-poor) likely lie side-by-side on the bluest sequence with $\mathrm{N}$-poor and C-normal stars (see Figs. 23 and 28 in Carretta et al. 2011).

The existence of a $\mathrm{Na}-\mathrm{O}$ anticorrelation in each population suggests that the classical chain of events (first generation, pollution, second generation, marking the birth of a genuine GC, as proposed in Carretta et al. 2010c) was present in each component. In turn, this may suggest a merger of two formerly individual clusters into an ancestral galaxy or two different, yet spatially close regions of star formation.

Villanova et al. (2010) found a different $\mathrm{N}$ content in the two group of stars with different $\mathrm{Ba}$ abundances. Campbell et al. (2012) recently studied the $\mathrm{CN}$ bandstrengths of a sample of 21 AGB stars and 17 RGB stars in NGC 1851; however, they were selected on the $V, B-V$ color-magnitude diagram, where the red and blue RGB sequence cannot be separated. In their two samples they found a quadrimodal distribution of $\mathrm{CN}$ strengths with the more $\mathrm{CN}$-weak populations coupled with low $\mathrm{Ba}$ abundances and the more $\mathrm{CN}$-strong peaks of the distributions associated with high $\mathrm{Ba}$ values.

Apart from these limited samples of RGB stars, no extensive spectroscopic analysis of the $\mathrm{N}$ abundance of objects on the reddest sequence was made up to now. With the present paper, we intend to fill this gap, deriving $\mathrm{N}$ estimates for about 60 red giants and providing another constraint for a better understanding of the complex star formation in NGC 1851.

\section{Observations and analysis}

The same observational data was recently used by us (Carretta et al. 2012a) to derive $\mathrm{Al}$ abundances for a large sample of RGB stars in NGC 1851: two exposures of $600 \mathrm{~s}$ each made with FLAMES-GIRAFFE mounted at VLT-UT2 and the highresolution grating $\operatorname{HR} 21(R=17300$, spectral range from $8484 \AA$ to $9001 \AA$ ). We refer to Carretta et al. (2012a) for details on the treatment of spectra (flat-fielding, calibration in wavelength, and sky subtraction). As before, atmospheric parameters (as well as abundances of $\mathrm{Fe}, \mathrm{O}, \mathrm{Na}, \mathrm{Mg}$, Ba etc.) were adopted from the highly homogeneous analysis by Carretta et al. (2011). The present sample is restricted to a magnitude range between $V \sim 17$ and $V \sim 15$.

In Carretta et al. (2012a), only a single feature was used to derive the $\mathrm{N}$ abundance, which, assuming $[\mathrm{C} / \mathrm{Fe}]=0$, reproduces the $\mathrm{CN}$ band strength to account for a possible contamination of Al lines. However, $\mathrm{N}$ lines are ubiquitous in the observed spectral range, and instead of treating them purely as contaminant, we can use them to derive $\mathrm{N}$ abundances for a large number of giants in NGC 1851. Our approach closely follows the one adopted in Carretta et al. (2012b) for the globular cluster M 4, which has a metallicity $[\mathrm{Fe} / \mathrm{H}]=-1.20 \mathrm{dex}$ (Carretta et al. 2009) similar to that of NGC $1851([\mathrm{Fe} / \mathrm{H}]=-1.16 \mathrm{dex})$. However, we remind the reader that the proprietary spectra taken in the case of $\mathrm{M} 4$ were acquired to maximise the signal around the $\mathrm{Al}$ lines, whereas the spectra with HR21 retrieved from the public ESO archive for NGC 1851 were observed in a public survey designed for other purposes and do not have an optimal $\mathrm{S} / \mathrm{N}$ for the task of abundance analysis for elements like $\mathrm{N}$.

To deal with this issue, we used an improved version of the procedure adopted in Carretta et al. (2012a). Briefly, 18 spectral regions dominated by $\mathrm{CN}$ bands and 8 regions that are apparently line-free were selected on a master spectrum obtained by summing up the ten spectra of giants with the best $\mathrm{S} / \mathrm{N}$ value. The list of these regions is given in Carretta et al. (2012b). For each $\mathrm{CN}$ feature, we measured the average flux within the in-line region, and this was normalized using a weighted reference continuum where the weights are equal to the width of the continuum regions. The $\mathrm{N}$ abundance from each feature was then obtained by comparing the normalized flux with the fluxes measured in the same way on three synthetic spectra computed using the Kurucz (1993) grid of model atmospheres (with the overshooting option switched on). The line list is described in Carretta et al. (2012a), and the synthetic spectra were computed with the package ROSA (Gratton 1988) by assuming $[\mathrm{C} / \mathrm{Fe}]=0.0 \mathrm{dex}$, three evenly spaced values of nitrogen, $[\mathrm{N} / \mathrm{Fe}]=-0.5,+0.0$, and $+0.5 \mathrm{dex}$, and the appropriate atmospheric parameters for each star.

Because of the non-optimal quality of the spectra, the final abundances of $\mathrm{N}$ were obtained after applying a severe $k \sigma$-clipping at $1.5 \sigma$ to the average abundance from individual features in each star. On average, only ten features survived this culling process. The average abundance ratio we found is $[\mathrm{N} / \mathrm{Fe}]=-0.082 \pm 0.037 \mathrm{dex}$ with $\mathrm{rms}=0.294 \mathrm{dex}$ from 62 stars.

As explained in Carretta et al. (2012a), an error due to flux measurement can be estimated for abundances derived through this procedure. This is obtained by estimating photometric errors from the $\mathrm{S} / \mathrm{N}$ of the spectra and from the width within each of the reference continuum and in-line regions (see details in Carretta et al. 2012a). The $\mathrm{N}$ abundances, derived by summing the original value of the $\mathrm{CN}$ band strength and its error, allow us to estimate the error associated to $\mathrm{N}$ abundances due to errors in the flux measurements. When we applied this procedure to a random $\mathrm{CN}$ feature used to estimate the $\mathrm{N}$ abundance, we found an average error of $+0.120 \pm 0.028$ dex from 62 stars in NGC 1851, which is a conservative estimate since the final $\mathrm{N}$ abundances rest on approximatively ten features per star. Actual star-to-star errors (those really relevant in the problem under scrutiny here) are then about 0.04 dex. Internal errors imputable to errors in the adopted atmospheric parameters have a similar impact, since star-to-star errors in effective temperature, gravity, model abundance and microturbulent velocity are only $4 \mathrm{~K}, 0.041 \mathrm{dex}, 0.034 \mathrm{dex}$, and $0.16 \mathrm{~km} \mathrm{~s}^{-1}$, respectively, 
Table 1. Abundance ratios [N/Fe] for RGB stars in NGC 1851.

\begin{tabular}{cccccccc}
\hline \hline Star & $\begin{array}{c}{[\mathrm{N} / \mathrm{Fe}]} \\
(\mathrm{dex})\end{array}$ & $\begin{array}{c}\mathrm{rms} \\
(\mathrm{dex})\end{array}$ & $\mathrm{Nr}^{1}$ & Star & $\begin{array}{c}{[\mathrm{N} / \mathrm{Fe}]} \\
(\mathrm{dex})\end{array}$ & $\begin{array}{c}\mathrm{rms} \\
(\mathrm{dex})\end{array}$ & $\mathrm{Nr}^{1}$ \\
\hline 13618 & -0.223 & 0.165 & 14 & 32256 & -0.195 & 0.134 & 11 \\
14827 & +0.032 & 0.424 & 10 & 35750 & -0.033 & 0.215 & 10 \\
16120 & -0.214 & 0.106 & 11 & 35999 & -0.031 & 0.125 & 7 \\
20189 & -0.064 & 0.231 & 9 & 36292 & +0.508 & 0.415 & 8 \\
20426 & -0.473 & 0.250 & 11 & 38484 & -0.201 & 0.236 & 14 \\
20653 & +0.227 & 0.119 & 9 & 38818 & +0.026 & 0.222 & 8 \\
20922 & -0.170 & 0.025 & 8 & 39364 & -0.204 & 0.255 & 16 \\
21453 & +0.052 & 0.040 & 8 & 40300 & -0.106 & 0.084 & 11 \\
21830 & +0.086 & 0.280 & 11 & 40615 & -0.035 & 0.033 & 7 \\
22360 & -0.146 & 0.243 & 11 & 41113 & -0.054 & 0.076 & 8 \\
22588 & -0.417 & 0.060 & 7 & 41855 & -0.387 & 0.500 & 9 \\
22813 & -0.310 & 0.158 & 12 & 43528 & +0.389 & 0.397 & 11 \\
23647 & -0.078 & 0.109 & 10 & 44224 & +0.092 & 0.665 & 12 \\
23765 & +0.161 & 0.081 & 11 & 44414 & -0.196 & 0.085 & 9 \\
25037 & -0.265 & 0.409 & 13 & 44939 & -0.264 & 0.060 & 11 \\
25497 & +0.173 & 0.190 & 8 & 45006 & -0.575 & 0.418 & 13 \\
25678 & -0.709 & 0.699 & 11 & 45090 & -0.197 & 0.256 & 13 \\
25799 & -0.570 & 0.278 & 10 & 45413 & +0.300 & 0.172 & 11 \\
26532 & +0.178 & 0.135 & 7 & 46228 & -0.195 & 0.146 & 12 \\
26552 & +0.035 & 0.123 & 10 & 46657 & +0.063 & 0.449 & 12 \\
26880 & +0.424 & 0.344 & 8 & 46958 & -0.462 & 0.124 & 10 \\
27491 & -0.402 & 0.089 & 7 & 47385 & -0.428 & 0.127 & 10 \\
28116 & +0.195 & 0.068 & 7 & 47795 & +0.380 & 0.198 & 13 \\
29203 & +0.313 & 0.161 & 13 & 48085 & -0.248 & 0.037 & 10 \\
29470 & +0.493 & 0.124 & 9 & 48277 & +0.212 & 0.238 & 8 \\
30286 & +0.150 & 0.116 & 8 & 48388 & +0.063 & 0.261 & 14 \\
31284 & -0.186 & 0.116 & 11 & 49965 & +0.177 & 0.133 & 8 \\
31399 & +0.317 & 0.247 & 9 & 50876 & -0.670 & 0.132 & 8 \\
31463 & -0.301 & 0.390 & 10 & 50973 & -0.330 & 0.071 & 9 \\
31520 & -0.002 & 0.162 & 9 & 51311 & -0.225 & 0.207 & 10 \\
32112 & +0.049 & 0.137 & 13 & 52570 & -0.639 & 0.700 & 11 \\
\hline & & & & & & &
\end{tabular}

Notes. ${ }^{(1)}$ Number of $\mathrm{CN}$ features used to derive $[\mathrm{N} / \mathrm{Fe}]$.

as estimated in Carretta et al. (2011). The total error expected in $\mathrm{N}$ abundance due to these uncertainties is 0.137 dex. However, these uncertainties have a negligible impact on our results, since they affect stars on both sequences.

Unfortunately, we have no stars in common with the small sample studied by Yong et al. (2009). In our sample, there are two stars in common with the analysis of Villanova et al. (2010), one in their Ba-poor group (star 35999) and the other in their Ba-rich group ( $\operatorname{star} 29203$ ). Taking into account the $\mathrm{C}$ abundance (assumed, in our case; derived, in Villanova et al. 2010), we found a difference (in the sense Villanova minus us) of 0.30 dex in $[\mathrm{N} / \mathrm{Fe}]$ for star 35999 and only 0.10 dex for star 29203. The comparison is not conclusive, owing to the different features and methods adopted in the two studies and resting on two objects only. For the aim of this analysis, the comparison of relative $\mathrm{N}$ abundances along the two sequences of the RGB, it is, however, the internal self-consistency that actually matters.

\section{Results and discussion}

The derived values of the $[\mathrm{N} / \mathrm{Fe}]$ ratios for the 62 stars in our sample are listed in Table 1, and their distribution is shown in Fig. 1.

As a working hypothesis, we defined the giants having $[\mathrm{N} / \mathrm{Fe}] \leq-0.15$ dex as $\mathrm{N}$-poor stars and those with $[\mathrm{N} / \mathrm{Fe}]>$ -0.15 dex as N-rich giants. The separating value simply corresponds to the dip in the histogram in Fig. 1.

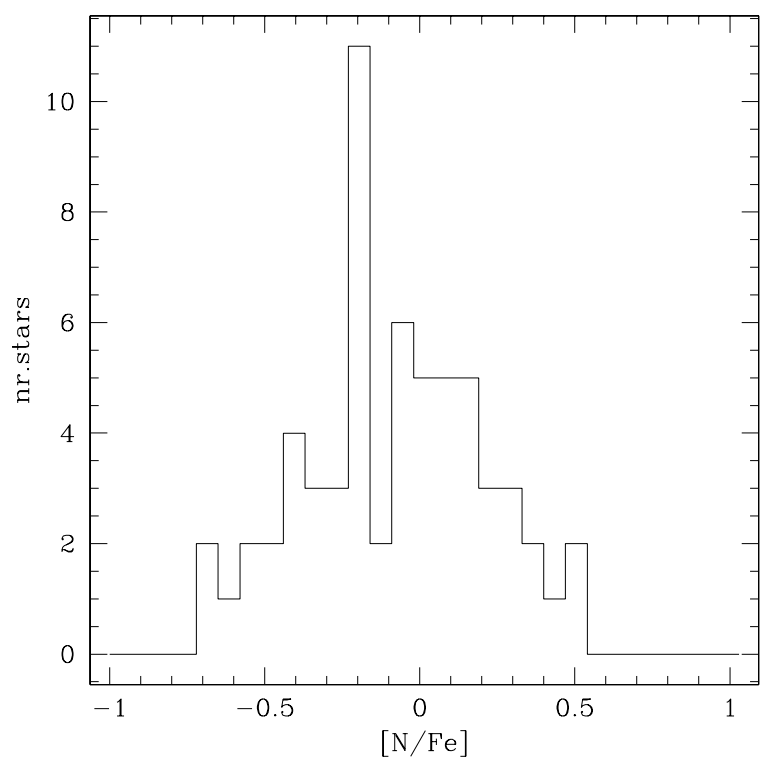

Fig. 1. Distribution of the $[\mathrm{N} / \mathrm{Fe}]$ ratios obtained among RGB stars in NGC 1851.

These two groups are indicated with different symbols in Fig. 2, where stars in our sample are plotted in the $y, u-y$ colourmagnitude diagram (CMD) using the unpublished Strömgren photometry kindly provided to us by Jae-Woo Lee (priv. comm.). 


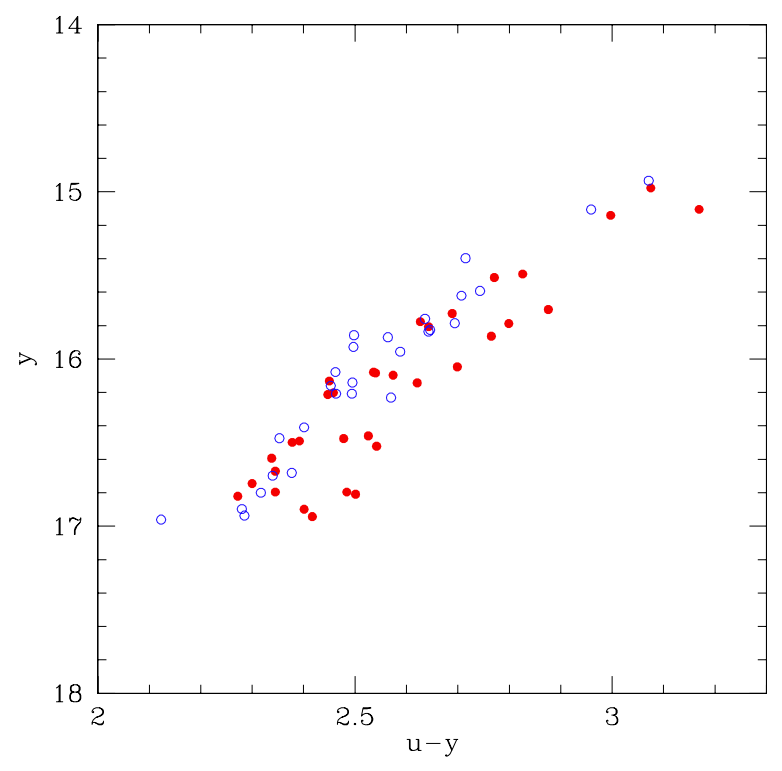

Fig. 2. Strömgren $y, u-y$ colour-magnitude diagram of RGB stars in our sample in NGC 1851 . Blue open circles are for giants with $[\mathrm{N} / \mathrm{Fe}] \leq$ -0.15 dex and filled red circles are for stars with $[\mathrm{N} / \mathrm{Fe}]>-0.15 \mathrm{dex}$.

From this CMD it is clear that all stars on the less populated, redder sequence are N-rich apart from a single outlier (possibly due to a combination of uncertainties in the photometry and/or $\mathrm{N}$ abundance). The bulk of giants, however, lie on the bluest RGB, which consists of a mix of N-rich and N-poor stars.

To represent this finding in a more quantitative way, we traced by eye the mean ridge line passing through the bluest sequence in the $y, u-y$ plane, using the N-poor stars as reference. Then, for each star, we computed the colour differences between the $u-y$ observed colour and the colour of the mean ridge line at the same magnitude $y$. The distributions of these residuals are plotted in Fig. 3 separately for the groups of N-rich (lower panel) and $\mathrm{N}$-poor (upper panel) giants.

The two distributions are clearly different, as confirmed by a statistical Kolmogorov-Smirnov test (see Fig. 4): the two populations have a very low probability $(0.0052)$ of being extracted from the same parent sample. Thus, the main conclusion of the present study is that the redder, less conspicous, secondary sequence on the RGB of NGC 1851 seems to be populated only by N-rich stars. The N-poor stars are almost completely segregated on the bluest part of the RGB with a fraction of N-rich giants.

There are six stars in common between our analysis and the sample of 17 RGB stars analyzed by Campbell et al. (2012). Using the Strömgren photometry available to us for these 6 objects, we see that the two stars with the highest values of $\delta \mathrm{S}(3839)$ (stars 41113 and 32112 in Table 1 of Campbell et al. 2012) lie on the redder RGB sequence. The other four stars $(46228,44414,47385$, and 44939) are located on the blue sequence and all have lower values of $\delta \mathrm{S}(3839)$.

In our study, we presented the first spectroscopic derivation of $\mathrm{N}$ abundances of giants in this cluster made for such a large number of stars on the RGB, which nicely supports the results by Villanova et al. (2010) and Campbell et al. (2012) based on much more limited samples. While we cannot state anything on the total $\mathrm{C}+\mathrm{N}+\mathrm{O}$ sum with not knowing the $\mathrm{C}$ abundance of the individual objects, we are able to spectroscopically ascertain that stars are located on the redder sequence in NGC 1851 because they are N-rich.

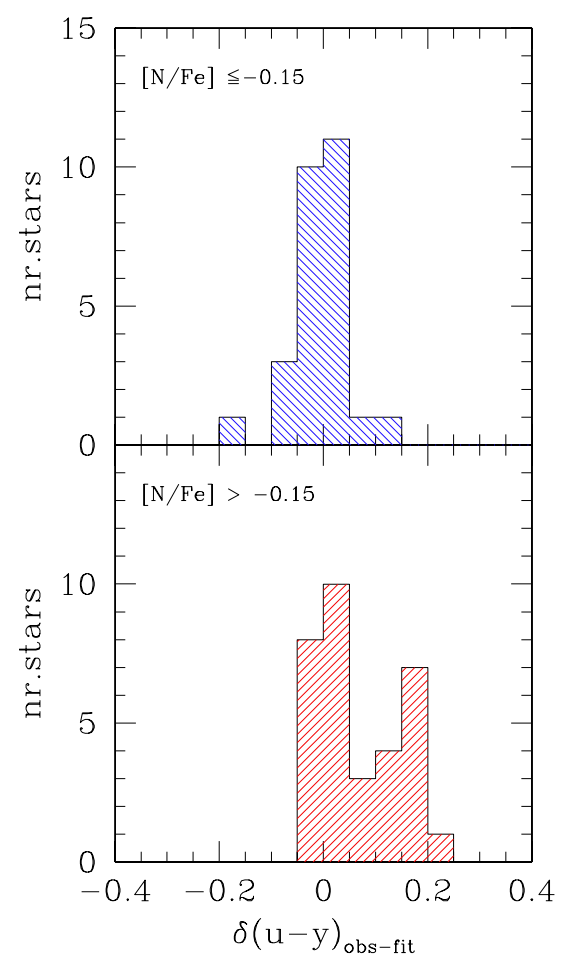

Fig. 3. Distribution of the differences $\delta(u-y)$ between the observed $u-y$ colour index of each star and the $u-y$ colour of the mean ridge line through the $\mathrm{N}$-poor stars at the same $y$ magnitude. The distribution is plotted separately for $\mathrm{N}$-poor giants and $\mathrm{N}$-rich stars (upper and lower panels, respectively).

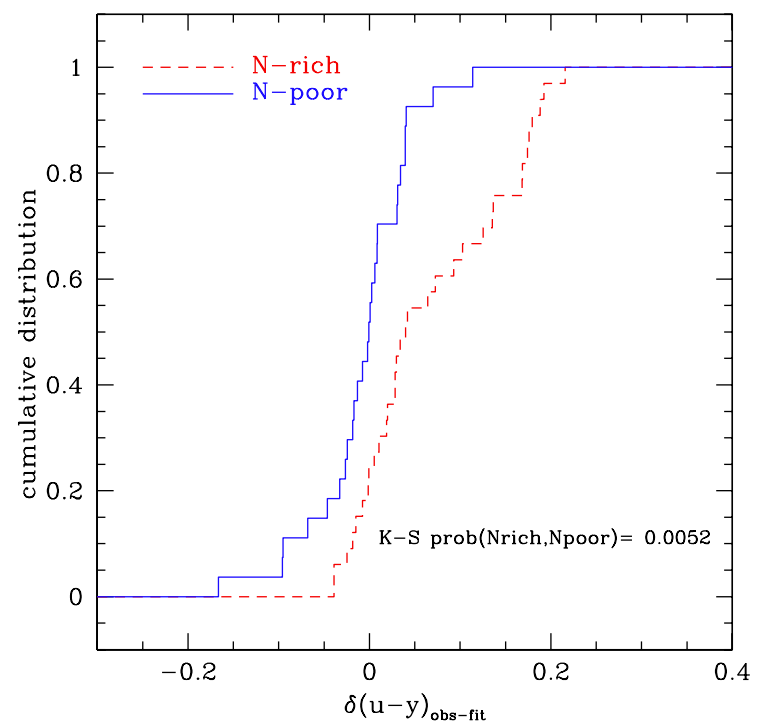

Fig. 4. Cumulative distributions of the residuals in $u-y$ colours between each star and the colour of the mean ridge line through N-poor stars. Solid line is for the distribution of $\mathrm{N}$-poor giants and dashed line for the $\mathrm{N}$-rich stars. The probability for the two distributions of being extracted from the same parent sample under the Kolmogorov-Smirnov statistical test is given.

Moreover, the present work strongly confirms the findings by Carretta et al. (2011) based on an independent approach. Simply from Strömgren photometry and explorative spectral synthesis of a C-rich/N-rich and of a C-rich/N-poor typical giant in NGC 1851 from Carretta et al. (2011), we concluded that stars on the redder sequence were "good candidate to be 
C-rich and N-rich (hence O-poor). On the other hand, the C-rich and $\mathrm{N}$-poor stars (i.e., O-rich) may not be separated from the other N-poor and C-normal stars in none of the Strömgren indices" (Carretta et al. 2011). In the present paper, we were able to confirm this statement by deriving directly the $N$ abundances of 62 RGB stars.

For 60 stars in our sample we have the values of the $h k$ index, kindly provided by Jae-Woo Lee (priv. comm.). The mean value of the index for stars on the redder sequence is +0.927 $(\mathrm{rms}=0.104)$, whereas we found a mean value of +0.799 $(\mathrm{rms}=0.114)$ for stars with normal $\mathrm{N}$ abundances. This difference can be compared to the full width at half-maximum of the RGB in NGC 1851 in $h k$ found by Lee et al. (2009b): 0.182 with a measurement error of 0.013 (their supplementary Table 3). A direct comparison to the $\mathrm{N}$ abundances found for BHB stars by Gratton et al. (2012c) is not easy because different abundance indicators (high-excitation lines) were used in that study, and nonLTE corrections are likely not negligible. Moreover, this comparison is hampered by the lack of knowledge of the absolute $\mathrm{C}$ values for our sample, where $\mathrm{N}$ abundances are derived from $\mathrm{CN}$ features. Finally, we found no evidence of radial variations in $\mathrm{N}$ abundances in the present sample.

Recently, Lardo et al. (2012) showed that the red sequence on the RGB of NGC 1851 is photometrically connected to the faint SGB discovered by Milone et al. (2008). This result, when coupled with evidence from spectroscopy in the present and previous studies (e.g. see Yong et al. 2009; Villanova et al. 2010 for a different view), gives support to the suggestion by Cassisi et al. (2008) that an increased amount of CNO (a factor about two) in stars of the faint SGB may explain the difference between the split sequences.

It is also well assessed (Villanova et al. 2010; Carretta et al. 2011; Campbell et al. 2012) that only Ba-rich stars are found on the redder sequence of the RGB with Ba-poor stars mostly (but not exclusively) confined to the more populated bluest part of the giant branch. This close similarity with the pattern found here for $\mathrm{N}$ abundances points towards the action of first generation polluters which experience episodes of third dredge-up, stars with initial mass in the range $1.5-3 M_{\odot}$. Moreover, Gratton et al. (2012b) investigated the abundance pattern of stars belonging to the two SGBs in NGC 1851; they found that stars on the faint SGB are $\mathrm{Sr}$ and $\mathrm{Ba}$-rich, while the group on the bright SGB show a low content of these elements. These findings point out that the observed spread of s-process elements, currently traced from the SGB up to the RGB, is real in this cluster.

On the other hand, the correlation between proton-capture elements (like $\mathrm{Na}$ and $\mathrm{Al}$ ) and the amount of s-process elements found by us (Carretta et al. 2011, 2012a) and other studies (Yong and Grundahl 2008; Villanova et al. 2010) presents a problem related to the different evolutionary times of different classes of first generation polluters. As discussed by Carretta et al. (2011), if the polluters were AGB stars, those producing proton-capture elements, such as $\mathrm{Al}$, are in the mass range between 4 and $8 M_{\odot}$. This leaves a time delay of about 200 Myr before the effects of mass loss from low-mass AGB stars (producing the bulk of s-process and enhanced CNO sum) may be incorporated in the gas forming stars of the second generation.

A possible solution of this puzzle is if the two sources of pollution are decoupled in origin. In other words, suppose that the gas enriched in s-process elements by low-mass AGB stars is not forged by stars of the forming proto-cluster. This gas may, for example, be collected in the potential well of a star-forming region immersed into a larger dwarf galaxy where matter leftover by a generation of low-mass AGB stars slowly inflows towards the denser proto-cluster regions. Here, this gas "unprocessed" by first generation cluster stars may combine with nuclearly processed ejecta and eventually form stars of a second generation that are rich in both $\mathrm{Al}$ and $\mathrm{N}$ and s-process elements. Such a variant to the first-generation-pollution-second generation sequence, where part of the pollution is not due to the same cluster stars, was originally introduced by Bekki et al. (2007).

As an alternative, if fast-rotating massive stars (Decressin et al. 2007) are responsible for the enrichment in proton-capture elements like $\mathrm{Na}$ and $\mathrm{Al}$, then the low-mass AGB stars within the first generation of cluster stars may contribute to the enrichment without any particular problem that is related to the age difference between intermediate and low-mass AGB stars.

Acknowledgements. V.D. is an ARC Super Science Fellow. This work was partially funded by the PRIN INAF 2011 grant "Multiple populations in globular clusters: their role in the Galaxy assembly" (PI E. Carretta), and the PRIN MIUR 2010-2011, "The Chemical and Dynamical Evolution of the Milky Way and Local Group Galaxies", (PI F. Matteucci), prot. 2010LY5N2T. We thank Šarūnas Mikolaitis for sharing with us the line list for CN provided by B. Plez, prof. Jae-Woo Lee for sharing his unpublished photometry, and Sandro Villanova for sending us C, N, O abundances and coordinates for individual stars of its sample. This research has made use of the SIMBAD database (in particular Vizier), operated at CDS, Strasbourg, France and of NASA's Astrophysical Data System.

\section{References}

Anderson, J. 1998, Ph.D. Thesis, Univ. California, Berkeley

Bekki, K., Campbell, S. W., Lattanzio, J. C., \& Norris, J. E. 2007, MNRAS, 377, 335

Carretta, E., Bragaglia, A., Gratton, R. G., D’Orazi, V., \& Lucatello, S. 2009, A\&A, 508, 695

Campbell, S. W., Yong, D., Wylie-De Boer, E. C., et al. 2012, ApJ, 761, L2

Carretta, E., Bragaglia, A., Gratton, R., et al. 2010a, ApJ, 712, L21

Carretta, E., Gratton, R. G., Lucatello, S., et al. 2010b, ApJ, 722, L1

Carretta, E., Bragaglia, A., Gratton, R. G., et al. 2010c, A\&A, 516, A55

Carretta, E., Lucatello, S., Gratton, R. G., Bragaglia, A., \& D’Orazi, V. 2011, A\&A, 533, A69

Carretta, E., D’Orazi, V., Gratton, R. G., \& Lucatello, S. 2012a, A\&A, 543, A117

Carretta, E., Gratton, R. G., Bragaglia, A., D’Orazi, V., \& Lucatello, S. 2012b, A\&A, 550, A34

Cassisi, S., Salaris, M., Pietrinferni, A., et al. 2008, ApJ, 672, L11

D’Antona, F., Bellazzini, M., Caloi, V., et al. 2005, ApJ, 631, 868

Decressin, T., Meynet, G., Charbonnel, C., Prantzos, N., \& Ekstrom, S. 2007, A\&A, 464, 1029

Gratton, R. G. 1988, Rome Obs. Preprint Ser., 29

Gratton, R. G., Sneden, C., \& Carretta, E. 2004, ARA\&A, 42, 385

Gratton, R. G., Carretta, E., \& Bragaglia, A. 2012a, A\&ARv, 20, 50

Gratton, R. G., Villanova, S., Lucatello, S., et al. 2012b, A\&A, 544, A12

Gratton, R. G., Lucatello, S., Carretta, E., et al. 2012c, A\&A, 539, A19

Han, S.-I., Lee, Y.-W., Joo, S.-J., et al. 2009, ApJ, 707, L190

Kurucz, R. L. 1993, CD-ROM 13 (Cambridge: Smithsonian Astrophysical Observatory)

Lardo, C., Milone, A. P., Marino, A. F., et al. 2012, A\&A, 541, A141

Lee, J.-W., Lee, J., Kang, Y.-W., et al. 2009a, ApJ, 695, L78

Lee, J.-W., Kim, S.-L., Lee, C.-U., \& Youn, J.-H. 2009b, Nature, 462, 480

Milone, A. P., Bedin, L., Piotto, G., et al. 2008, ApJ, 673, 241

Milone, A., Stetson, P. B., Piotto, G., et al. 2009, A\&A, 503, 755

Villanova, S., Geisler, D., \& Piotto, G. 2010, ApJ, 722, 118

Walker, A. R. 1992, PASP, 104, 1063

Yong, D., \& Grundahl, F. 2008, ApJ, 672, L29

Yong, D., Grundahl, F., D’Antona, F., et al. 2009, ApJ, 659, L62 